\title{
Treatment Outcome and Mortality Among Geriatric Patients Diagnosed with Multiple-Drug Resistant Tuberculosis: A Comparative Analysis from a Tertiary Referral Center
}

\author{
๑ Seda Tural Onur, @ Mediha Gonenc Ortakoylu, • Sinem Iliaz*, ๑ Figen Alkan \\ Yedikule Chest Diseases and Thoracic Surgery Training and Research Hospital, Clinic of Chest Diseases, Istanbul, Turkey \\ *Koc University Hospital, Clinic of Chest Diseases, Istanbul, Turkey
}

Abstract

Aim: Multidrug-resistant (MDR) tuberculosis (TB) and drug-sensitive-TB have long treatment periods which affect patient compliance and treatment outcomes. We planned to determine the factors that will affect the management of this disease in the elderly population.

Methods: In a period of two years, a total of 82 elderly patients with bacteriologically proven TB enrolled into our retrospective study between 2011-2018. The patients' demographic features, laboratory findings, and hospital records were analyzed.

Results: We enrolled 67 (87.7\%) patients with drug-sensitive TB and 15 (18.2\%) MDR-TB in our study. In the study population, 73.2\% $(n=60)$ were male and the mean age was $75 \pm 9$ years. When we compare treatment complications of MDR-TB and drug-sensitive-TB, we found out the rate of electrolyte imbalance, nephrotoxicity, and ocular toxicity more common among the MDR-TB group ( $p=0.008$, $p=0.008$, and $p=0.032$, respectively). When we compare the mortality rate, cure, and treatment success between MDR-TB and drugsensitive TB, there were no statistically significant results ( $p=0.898, p=0.549, p=0.488$; respectively).

Conclusion: However, we think that this was due to the low sample size. Nevertheless, we should be careful in terms of complications management of the geriatric population.

Keywords: Geriatric, mortality, pulmonary, tuberculosis, multidrug-resistant

\section{Introduction}

Tuberculosis (TB) continues to be a serious health problem with increasing human immunodeficiency virus infection, substance abuse, poverty, and immigration in our globalizing world. While there is a decrease in TB cases, mortality rates are still high. The average life expectancy is getting longer as the health care facilities increase in the world and as a result elderly population is increasing. TB is 30\% more frequent in elderly people in the United States (1). In spite of all difficulties, the world has done a great job in its struggle with TB. In addition to that, a new question arisen in the literature whether the geriatric population is a new sensitive group for TB treatment or not. The variability in clinical presentation and mortality rates in the elderly population differ from the young population. So these should be handled differently (2-4).

Both isoniazid and rifampicin resistance and rifampicin monoresistance (RR) are defined as multidrug resistance (MDR) according to the World Health Organization (5). MDR-TB patients' compliance and treatment outcomes are affected by potentially toxic drugs and longer treatment period compared to drug- sensitive TB (6-9). In elderly patients, polypharmacy due to comorbidities and physiological changes due to aging may make treatment management more difficult (10).

Besides the studies searching geographical and sociocultural differences in TB, there are small-scale studies on the elderly patients with TB in the literature.

Address for Correspondence: Seda Tural Onur, Yedikule Chest Diseases and Thoracic Surgery

Training and Research Hospital, Clinic of Chest Diseases, Istanbul, Turkey

E-mail: sedatural@yahoo.com ORCID: orcid.org/0000-0002-0657-0392

Received: 29.06.2021 Accepted: 04.10.2021

Copyright 2021 by The Medical Bulletin of istanbul Haseki Training and Research Hospital The Medical Bulletin of Haseki published by Galenos Yayinevi. 
In addition to the difficulties in the management of the patients with TB in itself, we evaluated the treatment results of the elderly patients in our TB clinic in order to determine the factors that would affect the management of the disease and to determine the measures to be taken in this direction.

\section{Methods}

\section{Study Design}

The study was undertaken in accordance with the principles of the Helsinki Declaration and had the approval of the institutional commitee. It was designed as a crosssectional study. The retrospective design study hasn't got local ethical approval however it has got hospital scientific data usage approval (no: E-66628377-929). A retrospective patient informed consent form is available.

\section{Patient Evaluation}

In our study, we enrolled elderly patients who were $\geq 65$ years old and hospitalized in drug-sensitive TB and MDR-TB clinics in two years of periods (24 months). Patients with at least two sputum smear positivity or any culture positivity for TB were diagnosed microbiologically as TB and they were classified according to the results of the drug susceptibility test (DST). We retrospectively recorded our patients' demographic data, clinical features, comorbidities, and laboratory results. The treatment complications of our patients who were diagnosed and treated by pulmonologists specialized on TB were recorded. Treatment and follow-up data were obtained from tuberculosis dispensary registries. Mortality data were obtained from the death notification system of the government database. Patients who were lost to follow-up were recorded as missing.

Hepatic toxicity was considered as an increase in transaminase value to 1.5 times than normal or basal value and/or the increase in bilirubin value above normal of the laboratory reference level. Nephrotoxicity was defined as serum creatinine value that was 1.5 times higher than the normal or basal value. According to the patient's baseline values, the electrolyte change that reached the lower and upper limits of the laboratory reference during the treatment was defined as the electrolyte imbalance. Endocrine dysfunction is defined as hypothyroidism not detected after TB drug use and evaluated by internal medicine specialist as not related to any other organic pathology. Gastrointestinal intolerance was recorded as stomach and bowel disorders not associated with hepatic dysfunction. Arthralgia was recorded as pain in at least one joint during the use of medical therapy. Skin rash was recorded as various skin reactions associated with drug use or drug allergy. Anemia, leukopenia, and thrombocytopenia associated with drug use were recorded as hematological disorders. Ototoxicity was recorded as a permanent auditory or vestibular abnormality associated with the use of aminoglycoside. Ocular toxicity was defined as impaired visual acuity or drug-related ocular pathology recognized by ophthalmologist. Complications of the central nervous system were defined as signs of systemic dysfunction such as dizziness, vertigo, insomnia, convulsion, and paralysis related to drug use.

\section{Statistical Analysis}

The Statistical Package for Social Sciences (SPSS) version 24.0 for Windows (IBM SPSS Statistics Data Editor) was used for statistical analysis of the data. Descriptive data were given as number of participants and frequency. Categorical variables were expressed as the number of patients and the percentage value. The comparison of categorical variables was performed using the chi-square and Fisher's Exact tests. Continuous variables were given as mean and standard deviation. The Shapiro-Wilk test was used to determine whether the continuous variables were normally distributed. For continuous variables, Student's t-test and Mann-Whitney $U$ test were used relative to the normality of distribution of the variables. A p-value of $<0.05$ was considered statistically significant.

\section{Results}

For two years follow-up periods, 82 patients with $\geq 65$ years of age who were hospitalized in the drug-sensitive TB and MDR-TB clinics were included in our study. The mean age of our patients was 75 \pm 9 years (range 65-94 years). According to the DST results, $18.2 \%(n=13)$ of our cases were MDR-TB and $81.7 \%(n=64)$ were drugsensitive TB (Table 1). Among the study population, $26 \%$ $(n=20)$ of our cases had a prior history of- TB. Of the cases, $89.6 \%(n=69)$ were smear-positive pulmonary TB (For the distribution of TB involvement site go to Figure 1). The mean follow-up period was $810 \pm 525$ days and 8 patients $(10.4 \%)$ were loss to follow-up. The rate of comorbid diseases in our study population was $68.8 \%$. When we compared the patients with comorbidities, there were no significant differences between the patients with the MDR and sensitive TB in terms of treatment success, rate of mortality, and treatment adverse effects ( $p>0.05$ for all parameters).

According to DST results, our patients had 19.5\% isoniazid, $16.9 \%$ rifampicin, $11.7 \%$ ethambutol, $11.7 \%$ streptomycin, and $1.3 \%$ quinolone resistance. In the comparison of MDR and drug-sensitive TB patients, the MDR-TB group had higher electrolyte disturbance, nephrotoxicity, and visual impairment during the treatment period ( $p=0.02, p=0.02$, and $p=0.03$, respectively). Besides, hypothyroidism, psychosis, ototoxicity, and neuropathy 
were more common in the MDR-TB group, the difference was not statistically significant ( $p>0.05$ ) (Table 1).

When treatment success was evaluated, it resulted in $53.6 \%$ cure. Treatment failure was present in 1 case (1.3\%) and treatment abandonment was 6 (7.8\%). During followup, $9(11.7 \%)$ cases were exitus during hospitalization and $13(16.9 \%)$ patients were exitus during follow-up as outpatient. In the comparison of the TB groups, no statistically significant difference was found between mortality, cure, treatment success, and abandonment of treatment ( $p=0.89, p=0.55, p=0.49, p=0.27$, respectively).

\begin{tabular}{|c|c|c|c|}
\hline & $\begin{array}{l}\text { Drug sensitive TB, } \\
\text { n (\%) }\end{array}$ & $\begin{array}{l}\text { Multidrug } \\
\text { resistant TB, } \\
\text { n (\%) }\end{array}$ & $\mathbf{p}^{*}$ \\
\hline Electrolyte imbalance & $9(14.1 \%)$ & $6(46.2 \%)$ & $0.02^{*}$ \\
\hline Nephrotoxicity & $9(14.1 \%)$ & $6(46.2 \%)$ & $0.02^{*}$ \\
\hline Ototoxicity & $1(1.6 \%)$ & $2(15.4 \%)$ & 0.07 \\
\hline Ocular toxicity & $2(3.1 \%)$ & $3(23.1 \%)$ & $0.03^{*}$ \\
\hline Gastric intolerance & $25(39.1 \%)$ & $6(46.2 \%)$ & 0.64 \\
\hline Psychosis & $3(4.7 \%)$ & $2(15.4 \%)$ & 0.20 \\
\hline Depression & $2(3.1 \%)$ & $0(0 \%)$ & $>0.99$ \\
\hline Artralgia & $1(1.6 \%)$ & $1(7.7 \%)$ & 0.31 \\
\hline Hepatotoxicity & $38(59.4 \%)$ & $4(30.8 \%)$ & 0.06 \\
\hline Skin rash & $10(15.6 \%)$ & $0(0 \%)$ & 0.20 \\
\hline Convulsion & $1(1.6 \%)$ & $1(7.7 \%)$ & 0.31 \\
\hline Vertigo & $4(6.3 \%)$ & $1(7.7 \%)$ & $>0.99$ \\
\hline Neuropathy & $4(6.3 \%)$ & $3(23.1 \%)$ & 0.09 \\
\hline Hypothyroidism & $1(1.6 \%)$ & $2(15.4 \%)$ & 0.07 \\
\hline
\end{tabular}

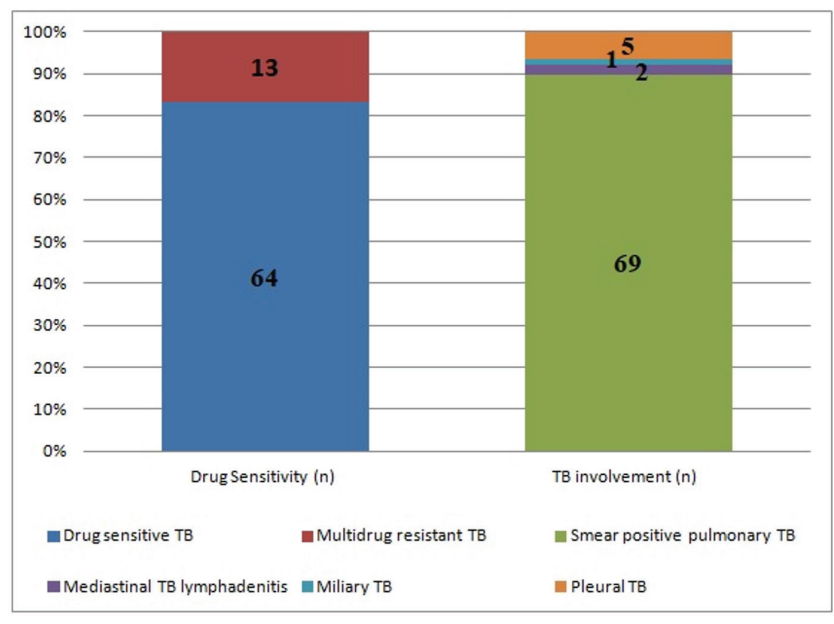

Figure 1. Distribution of the patients according to $\mathrm{TB}+$ involvement

TB: Tuberculosis

\section{Discussion}

When the results of TB treatment were evaluated in elderly patients, there were limited number of studies in the literature. According to these data, the mortality rate of TB in elderly is increased compared to the younger population (11). While treatment results about drug-sensitive TB and MDR-TB were available in younger population, we wondered about the treatment results and the factors affecting that in the elderly. There were no differences in treatment success and cure rates between drug-sensitive TB and MDR-TB in our study. However, we found out that the treatment complications rates are increased in MDR-TB in the elderly.

In studies from Far Eastern countries, the mortality rate in the presence of TB in the elderly was reported $3.9 \%$ higher than the general population (12). Increased mortality in the elderly is associated with reduced immunity and increased comorbidities. Although TB susceptibility of this population increases, there are also delays in diagnosis due to different clinical presentations in this group of patients $(5,13)$. Moreover, low body mass index, smoking history, and poorly controlled diabetes increase TB susceptibility in elderly (14-17). TB frequency is increased in impaired nutritional status (18). Because of all these, we aimed to increase the awareness of clinicians on this issue by our study.

It is mentioned that the incidence of drug side effects in the treatment process of elderly TB patients with comorbidities is high in the literature (19). The most serious side effect is reported as hepatotoxicity (20). Hepatotoxicity is frequently associated with isoniazid, rifampin, and pyrazinamide, while follow-up of medical therapy requires precision. We reported that patients who were on MDR-TB treatment were found to be more sensitive to drug side effects compared to the patients with drug-sensitive TB. We found out that the most common adverse effects of anti-TB treatment were hepatotoxicity and gastric intolerance. In addition to that, clinicians should be more careful to detect and manage nephrotoxicity and electrolyte imbalance, because these are common and may affect the vital functions of the patients.

In the literature, it was found that radiological patterns could be a sign of mortality in TB patients, but we could not find any relation in the elderly in our study. We think that it might be due to our small sample size (11). Another possible reason is that, besides the non-specific clinical presentations in elderly patients, also radiological patterns may not be guiding for the prognosis. For instance, decreased appetite decreased daily functional activity, and subfebrile fever may occur frequently in elderly patients (21). 


\section{Study Limitations}

Because our study was designed to be retrospective, our sample size was not so large. The drug history of patients after discharge could not be questioned and this information could not be accessed from the system. This was missing data in our study. Another limitation is the scarce diversity of ICD codes related to mortality which was accessed from the electronic mortality system. Despite the small number of geriatric MDR-TBC patients in the community, our study is a valuable study because it has a sufficient number of cases, is one of the few studies conducted in the geriatric group, and contributes to the literature.

\section{Conclusion}

The results of treatment success, cure, and mortality rates of drug-sensitive TB and MDR-TB in the geriatric population were not different. However, it is recommended to be more careful in terms of drug side effect management and treatment interventions in MDR-TB patients with increased fragility. Especially, close monitoring of electrolyte and liver function tests and periodic eye examination may facilitate the management of complications by clinicians. Further studies are needed to generalize our results.

\section{Authorship Contributions}

Concept: M.G.O., S.T.O., Design: M.G.O., S.T.O., Data Collection or Processing: F.A., M.G.O., S.T.O., Analysis or Interpretation: S.T.O., S.I., Literature Search: S.T.O., F.A., S.I., Writing: S.T.O., S.I.

Conflict of Interest: No conflict of interest was declared by the authors.

Financial Disclosure: The authors declared that this study received no financial support.

\section{References}

1. Pratt RH, Winston CA, Kammerer JS, Armstrong LR. Tuberculosis in older adults in the United States, 1993-2008. J Am Geriatr Soc 2011;59:851-7.

2. Morris CD. Pulmonary tuberculosis in the elderly: a different disease? Thorax 1990;45:912-3.

3. Schaaf HS, Collins A, Bekker A, Davies PD. Tuberculosis at extremes of age. Respirology 2010;15:747-63.

4. Dousa KM, Kurz SG, Bark CM, Bonomo RA, Furin JJ. DrugResistant Tuberculosis: A Glance at Progress and Global Challenges. Infect Dis Clin North Am 2020;34:863-86.

5. World Health Organization Report. Global tuberculosis control 2017. Geneva: World Health Organization (WHO/HTM/ TB/2017.23); 2017. https://www.who.int/tb/publications/ global_report/gtbr2017_main_text.pdf (Accessed March 2019).
6. Diel R, Rutz S, Castell S, Schaberg T. Tuberculosis: cost of illness in Germany. Eur Respir J 2012;40:143-51.

7. Loddenkemper R, Sotgiu G, Mitnick CD. Cost of tuberculosis in the era of multidrug resistance: will it become unaffordable? Eur Respir J 2012;40:9-11.

8. Natarajan A, Beena PM, Devnikar AV, Mali S. A systemic review on tuberculosis. Indian J Tuberc 2020;67:295-311.

9. Singh R, Dwivedi SP, Gaharwar US, Meena R, Rajamani P, Prasad T. Recent updates on drug resistance in Mycobacterium tuberculosis. J Appl Microbiol 2020;128:1547-67.

10. Byng-Maddick R, Noursadeghi M. Does tuberculosis threaten our ageing populations? BMC Infect Dis 2016;16:119.

11. Yen YF, Feng JY, Pan SW, Chuang PH, Su VY, Su WJ. Determinants of mortality in elderly patients with tuberculosis: a populationbased follow-up study. Epidemiol Infect 2017;145:1374-81.

12. Ministry of Health and Welfare. Vital Statistics in Taiwan. Taiwan: Ministry of Health and Welfare, Executive Yuan. (http://www.mohw.gov.tw/CHT/DOS/ Statistic.aspx?f_list_ no=312\&fod_list_no=1601). (Accessed December 2016)

13. Storla DG, Yimer S, Bjune GA. A systematic review of delay in the diagnosis and treatment of tuberculosis. BMC Public Health 2008;8:15.

14. Leung CC, Yew WW, Chan CK, et al. Tuberculosis in older people: a retrospective and comparative study from Hong Kong. J Am Geriatr Soc 2002;50:1219-26.

15. Leung CC, Lam TH, Chan WM, et al. Lower risk of tuberculosis in obesity. Arch Intern Med 2007;167:1297-304.

16. Leung CC, Lam TH, Chan WM, et al. Diabetic control and risk of tuberculosis: a cohort study. Am J Epidemiol 2008; 167:1486-94.

17. Mirzayev F, Viney K, Linh NN, et al. World Health Organization recommendations on the treatment of drug-resistant tuberculosis, 2020 update. Eur Respir J 2021;57:2003300.

18. Donald PR, Marais BJ, Barry CE 3rd. Age and the epidemiology and pathogenesis of tuberculosis. Lancet 2010;375:1852-4.

19. Umeki S. Age-related changes in the manifestations of tuberculosis. Implications for drug therapy. Drugs Aging 1991;1:440-57.

20. Woo J, Chan HS. Therapeutic problems in the management of elderly patients with tuberculosis. Adverse Drug React Toxicol Rev 1992;11:13-8.

21. Ijaz K, Dillaha JA, Yang Z, Cave MD, Bates JH. Unrecognized tuberculosis in a nursing home causing death with spread of tuberculosis to the community. I Am Geriatr Soc 2002;50:1213-8. 